\title{
BMJ Open Atrial fibrillation in patients with severe mental disorders and the risk of stroke, fatal thromboembolic events and bleeding: a nationwide cohort study
}

\author{
Mette Søgaard, ${ }^{1,2}$ Flemming Skjøth,, ${ }^{2,3}$ Jette Nordstrøm Kjældgaard, , \\ Torben Bjerregaard Larsen, ${ }^{1,2,4}$ Søren Pihlkjær Hjortshøj, ${ }^{4,5}$ Sam Riahi ${ }^{1,5,4}$
}

\section{To cite: Søgaard M, Skjøth F, Kjældgaard JN, et al. Atrial fibrillation in patients with severe mental disorders and the risk of stroke, fatal thromboembolic events and bleeding: a nationwide cohort study. BMJ Open 2017;7:e018209. doi:10.1136/ bmjopen-2017-018209 \\ - Prepublication history andadditional material for thispaper are available online. To view these files, please visit the journal online (http://dx.doi. org/10.1136/bmjopen-2017- 018209).}

Received 14 June 2017 Revised 4 October 2017 Accepted 5 October 2017

\section{(a) CrossMark}

${ }^{1}$ Department of Cardiology, Aalborg University Hospital, Aalborg, Denmark

${ }^{2}$ Aalborg Thrombosis Research Unit, Department of Clinical Medicine, Faculty of Health, Aalborg University, Aalborg, Denmark

${ }^{3}$ Unit for Clinical Biostatistics, Aalborg University Hospital, Aalborg, Denmark ${ }^{4}$ AF Study Group, Aalborg University Hospital, Aalborg, Denmark

${ }^{5}$ Department of Clinical Medicine, Aalborg University, Aalborg, Denmark

Correspondence to Dr Mette Søgaard; mette.soegaard@rn.dk

\section{ABSTRACT}

Objectives Outcomes of atrial fibrillation (AF) in patients with severe mental disorders are largely unknown. We compared rates of stroke, fatal thromboembolic events and bleeding in patients with AF with and without mental disorders.

Design Nationwide registry-based cohort study. Setting Denmark (population 5.6 million), 2000-2015. Participants Patients with AF with schizophrenia $(n=534)$, severe depression $(n=400)$ or bipolar disease $(n=569)$ matched 1:5 on age, sex and calendar time to patients with AF without mental disorders.

Exposure Inpatient or hospital-based outpatient diagnosis of schizophrenia, severe depression or bipolar disease.

Primary and secondary outcome measures HRs for stroke, fatal thromboembolic events and major bleeding comparing patients with and without mental disorders estimated by Cox regression with sequential adjustment for risk factors for stroke and bleeding, comorbidity and initiation of oral anticoagulant therapy (OAT).

Results Compared with matched comparisons, crude 5-year HRs of ischaemic stroke were 1.37 (95\% Cl 0.88 to 2.14 ) for schizophrenia, 1.36 ( $95 \% \mathrm{Cl} 0.89$ to 2.08 ) for depression and 1.04 (95\% $\mathrm{Cl} 0.69$ to 1.56) for bipolar disease. After adjusting for risk factors, comorbidity and OAT, these HRs declined towards the null. Crude HRs of fatal thromboembolic events were $3.16(95 \% \mathrm{Cl} 1.78$ to 5.61) for schizophrenia, 1.31 (95\% $\mathrm{Cl} 0.67$ to 2.56$)$ for depression and 1.53 (95\% $\mathrm{Cl} 0.93$ to 2.53$)$ for bipolar disease. Rates of major bleeding were increased in patients with schizophrenia (crude HR 1.37, 95\% Cl 0.99 to 1.90 ) and severe depression (HR 1.25, 95\% Cl 0.87 to 1.78) but not bipolar disease (HR $0.82,95 \% \mathrm{Cl} 0.58$ to 1.15).

Conclusion Patients with AF with schizophrenia or severe depression experienced increased rates of stroke and major bleeding compared with matched comparisons. This increase was largely explained by differences in the prevalence of risk factors for stroke and bleeding, comorbidity and initiation of OAT during follow-up. Patients with AF with schizophrenia further experienced higher mortality following thromboembolic events than matched comparisons without mental disorders.

\section{Strengths and limitations of this study}

- The study included all patients with a hospital diagnosis of atrial fibrillation in Denmark in 2000-2015. The study had complete follow-up on all participants from the nationwide Danish Civil Registration System.

The study was conducted in a government-financed healthcare system with equal access for the entire Danish population.

- Despite equal access to tax-supported healthcare in Denmark, diagnoses in patients with mental disorders may have been under-reported.

- The study lacked data on alcohol consumption, smoking, exercise and other lifestyle-related risk factors associated with increased risk of study outcomes. We were able to adjust for hospital diagnoses of alcohol-related conditions and other lifestyle-related diseases, but cannot exclude residual confounding.

- Finally, the data did not contain information on quality and compliance with oral anticoagulant therapy.

\section{INTRODUCTION}

Cardiovascular diseases are highly prevalent in patients with severe mental disorders such as schizophrenia, bipolar disease and severe depression, ${ }^{1}$ contributing to a shorter life expectancy of 10-20 years than the general population. ${ }^{2}$ Potential explanations include a high prevalence of cardiovascular risk factors such as smoking, dyslipidaemia, hypertension, diabetes and obesity. ${ }^{3-5}$ In addition, antipsychotic medications may adversely affect cardiovascular disease risk via metabolic pathways involving dyslipidaemia, weight gain and diabetes. $^{67}$

Atrial fibrillation (AF) is the most common cardiac arrhythmia, affecting up to $1 \%-2 \%$ of the population in developed countries, and confers a substantial increased risk of stroke, heart failure and death. ${ }^{8}$ Despite increasing 
clinical and research focus on cardiovascular diseases in patients with AF with mental disorders, the stroke risk in patients with mental disorders is largely unknown. Prior studies have shown that patients with AF with mental disorders are less likely to start oral anticoagulant therapy (OAT) than those without, ${ }^{910}$ and that those who receive OAT have worse anticoagulation control ${ }^{91112}$ and increased risk of major haemorrhage. ${ }^{11}{ }^{13}$ However, these studies only assessed outcomes in patients receiving OAT for at least 100-180 days, excluding patients who never initiated therapy and those who discontinued therapy shortly after initiation. ${ }^{11} 13$

We aimed to examine the prognostic importance of severe mental disorders in patients with AF. In a nationwide cohort of patients with incident $\mathrm{AF}$, we conceived a matched cohort study to compare the risk of stroke, fatal thromboembolic events and major bleeding in patients with $\mathrm{AF}$ with a prior diagnosis of schizophrenia, severe depression or bipolar disease with matched comparison cohorts without these disorders. By sequentially adjusting for stroke risk factors, bleeding risk factors, comorbidity and use of OAT, we sought to further characterise the association between mental disorders and AF outcomes.

\section{METHODS}

\section{Data sources}

This cohort study linked three well-established Danish registries nationwide: the National Patient Register, ${ }^{14}$ the National Prescription Registry ${ }^{15}$ and the Civil Registration System. ${ }^{16}$ The National Patient Register holds information on dates of admission and discharge, and discharge diagnoses classified according to the International Classification of Diseases for more than 99\% of hospital admissions in Denmark. The National Prescription Registry contains data on all prescription purchases by Danish residents since 1995. Data include patients' civil registration number, date of dispensing, and type and quantity of drug prescribed. The Danish Person Registry holds data on sex, date of birth, vital and emigration status. Online supplementary table 1 provides information on codes for all diagnoses and medications. The registries were linked using the unique personal civil registration number assigned to all Danish residents, allowing a true population-based study covering all 5.6 million inhabitants of Denmark during the study period. We performed all linkages within Statistics Denmark, a governmental institution that collects and processes information for statistical and scientific purposes. ${ }^{17}$

\section{Study population}

We established a cohort of all patients with incident non-valvular AF, defining non-valvular $\mathrm{AF}$ as the presence of AF, and baseline absence of mitral stenosis or mechanical heart valves. Specifically, we identified all patients discharged with a first hospital diagnosis of non-valvular AF between 2000 and 2015. To ensure sufficient clinical record history for treatment and diagnoses, we excluded patients who had not been residents in Denmark for at least 1 year before date of AF diagnosis (index date). We further excluded patients with valvular AF; patients who died on the day of AF diagnosis; and patients with a fatal thromboembolic event defined as death within the following 30 days of ischaemic stroke, systemic embolism, pulmonary embolism or myocardial infarction before AF diagnosis (online supplementary figure 1). The positive predictive value (PPV) of an $\mathrm{AF}$ diagnosis in the National Patient Register is $95 \%$ (95\% CI 89 to 98$).{ }^{18}$

\section{Exposure}

Through the National Patient Register, we identified all patients in the study population with an inpatient or outpatient diagnosis of schizophrenia, bipolar disease or severe depression before the index date. In Denmark, these mental disorders are primarily treated in public hospitals ensuring a high coverage of contacts with psychiatric disorders. The PPV of a diagnosis of mental disorders in the National Patient Register is 98\% $(95 \%$ CI 90 to 99$).{ }^{18}$

To control for confounding by reducing imbalance in the data and thereby model dependence and bias, we used coarsened exact matching to produce a one-to-five match of patients with schizophrenia, severe depression or bipolar disease on age, sex and calendar time (year of index date) to comparison cohorts of patients with $\mathrm{AF}$ without mental disorders. ${ }^{19}$ Patients' age was grouped in approximately 5-year intervals based on the statistical software package Stata V.14 function cem's algorithm for automatic coarsening. ${ }^{20}$ To evaluate the effect of matching on the balance between baseline variables, we estimated the absolute standardised differences before and after matching.

\section{Patient characteristics}

Baseline comorbidity was defined according to medication claims within the year before the AF diagnosis and/ or history of primary or secondary hospital discharge diagnoses (excluding emergency room diagnoses) since 1994. Comorbidity information included cardiovascular and metabolic diseases, and lifestyle-related diseases (eg, alcohol-related diseases such as alcoholic liver disease, and alcoholic polyneuropathy, cardiomyopathy, gastritis or myopathy, and alcohol-induced pancreatitis). We further combined baseline information into the $\mathrm{CHA}_{2} \mathrm{DS}_{2}$-VASc stroke risk score (congestive heart failure; hypertension; age $\geq 75$ years; diabetes mellitus; prior stroke, transient ischemic attack or thromboembolism; vascular disease, age 65-74 years; female sex) ${ }^{21}$ to summarise perceived stroke risk at baseline, and the hypertension; abnormal renal function; abnormal hepatic function; prior stroke; bleeding; labile INR (not included); elderly age (aspirin, clopidogrel or non-steroidal anti-inflammatory drugs; alcohol intake (HAS-BLED score ${ }^{22}$ as a measure of bleeding risk at baseline (see score definitions in online supplementary table 2). 


\section{Outcomes}

Study outcomes were ischaemic stroke, fatal thromboembolic events of ischaemic stroke, systemic embolism, pulmonary embolism or myocardial infarction (as defined above), and major bleeding events recorded as intracranial, gastrointestinal, and major bleeding in various anatomical positions and reported in total as 'any bleeding'. We derived all outcomes from hospital diagnoses in the National Patient Register. Stroke diagnoses were required to be primary in-hospital codes, excluding emergency room and ambulatory diagnoses, to ensure higher validity.

\section{Statistical analyses}

We followed all patients from their AF diagnosis and up to 5 years after baseline. Follow-up was censored at time of death, migration, study end (31 December 2015) or the outcome of interest, whichever came first. Patient baseline characteristics were presented as proportions for discrete variables and means with $\mathrm{SD}$ for continuous variables. Crude incidence rates were calculated as the number of events divided by person-time. Cumulative incidence functions (by means of the Aalen-Johansen estimator), assuming death as competing risk, were used to depict risk of outcome during follow-up. We assessed the association between each mental disorder and study outcome using Cox proportional hazard regression with stratification on the matched groups. To assess to which extent the observed association could be explained by comorbidity and/or use of OAT, we performed sequential cumulative adjustment for (1) stroke risk and bleeding risk as summarised by the components of the $\mathrm{CHA}_{2} \mathrm{DS}_{2}$-VASc and HAS-BLED scores , and comorbidities not included in $\mathrm{CHA}_{2} \mathrm{DS}_{2}$-VASc and HAS-BLED (chronic pulmonary disease, cancer and venous thromboembolism); and (2) use of OAT during follow-up, modelled as a timevarying covariate shifting from untreated to treated status at first observed prescription of any oral anticoagulant (OAC) treatment. We excluded age and sex in the $\mathrm{CHA}_{2} \mathrm{DS}_{2}-\mathrm{VASc}$ and HAS-BLED scores as these were included as matching factors and perfectly balanced between comparison cohorts (online supplementary table 3). The distribution of time to OAC treatment initiation was presented by cumulative incidence curves (online supplementary figure 2). Point estimates were reported with $95 \%$ CIs and a p value less than 0.05 was considered statistically significant.

\section{RESULTS}

We identified 260974 patients with AF during the study period. After exclusions, the study cohort comprised 253 741 patients with $\mathrm{AF}$, of whom 534 patients had schizophrenia, 400 had severe depression and 569 had bipolar disease (online supplementary figure 1). Table 1 shows baseline characteristics according to the presence and type of mental disorder. Patients with AF with schizophrenia were substantially younger (mean age 64.5 years), whereas the age of patients with severe depression (73.7 years) and bipolar disease (73.0 years) was comparable with patients without mental disorders (73.3 years). Baseline stroke risk appeared lower in patients with schizophrenia; the mean $\mathrm{CHA}_{2} \mathrm{DS}_{2}$-VASc score was 2.5 vs 3.1 in patients without mental disorders. However, this was explained by the lower age of patients with schizophrenia. The $\mathrm{CHA}_{2} \mathrm{DS}_{2}$ VASc score was 3.6 in patients with severe depression and 3.3 in patients with bipolar disease (table 1). The higher $\mathrm{CHA}_{2} \mathrm{DS}_{2}$-VASc score in patients with severe depression was primarily driven by a large proportion of women $(61.8 \%$ vs $46.7 \%$ in comparisons) and patients with prior stroke $(30.3 \%$ vs $16.9 \%)$. In comparison, $14.2 \%$ of patients with schizophrenia had prior stroke; they also had lower prevalence of hypertension, myocardial infarction and peripheral arterial disease. Compared with patients with no mental disorder, the HAS-BLED score was also higher in patients with severe depression and bipolar disease, whereas it was lower in patients with schizophrenia. Alcohol-related diseases were prevalent across all mental disorders, particularly for patients with schizophrenia or bipolar disease ( $20 \%$ vs $4 \%$ in comparisons). Online supplementary table 3 shows the characteristics of patients with schizophrenia, severe depression and bipolar disease and their matched comparisons. After matching, the mean $\mathrm{CHA}_{2} \mathrm{DS}_{2}-\mathrm{VASc}$ score was 2.3 in patients with $\mathrm{AF}$ without schizophrenia (online supplementary table 3).

\section{Ischaemic stroke}

Figure 1 displays cumulative incidence curves for ischaemic stroke in the matched cohorts. The rate of ischaemic stroke at 5 years was 1.96 events per 100 person-years in patients with AF with schizophrenia vs 1.30 in matched comparisons (table 2), yielding a crude HR of 1.37 (95\% CI 0.88 to 2.14) with CIs including unity (figure 2). After adjustment for stroke risk as summarised by the $\mathrm{CHA}_{2} \mathrm{DS}_{2}$-VASc and HAS-BLED scores and other comorbidities, this HR was 1.29 (95\% CI 0.81 to 2.07 ) (figure 2). The rate of OAT initiation in patients with AF with mental disorders was substantially lower than in matched comparisons (online supplementary figure 2), and after additional adjustment for OAT use during follow-up the HR of ischaemic stroke was $1.16 \quad(95 \%$ CI 0.72 to 1.87 ) (figure 2 ). At 5 years the rate of ischaemic stroke was 2.74 per 100 person-years in patients with severe depression vs 1.93 in matched comparisons (crude HR 1.36, 95\% CI 0.89 to 2.08). Similar to patients with schizophrenia, the crude HR was substantially attenuated by sequential adjustment for stroke risk factors, comorbidity and OAT (HR 1.01, 95\% CI 0.64 to 1.58) (figure 2). This pattern of diminishing HRs with sequential adjustment for stroke risk factors and OAT was also evident in patients with bipolar disease in whom the crude HR was 1.04 (95\% CI 0.69 to 1.56) and the fully adjusted HR was 0.85 (95\% CI 0.55 to 1.29$)$. Thus, compared with patients with AF without mental disorders, the fully adjusted HRs indicated comparable HRs of ischaemic stroke across all mental disorders with wide CIs that crossed unity (figure 2). 
Table 1 Descriptive characteristics of patients with incident atrial fibrillation in Denmark according to presence of mental health disorders before matching

\begin{tabular}{|c|c|c|c|c|}
\hline Characteristics, \% (n) & $\begin{array}{l}\text { No mental } \\
\text { disorder } \\
(\mathrm{n}=252238)\end{array}$ & $\begin{array}{l}\text { Schizophrenia } \\
(n=534)\end{array}$ & $\begin{array}{l}\text { Severe } \\
\text { depression } \\
(n=400)\end{array}$ & $\begin{array}{l}\text { Bipolar disease } \\
(\mathrm{n}=569)\end{array}$ \\
\hline \multicolumn{5}{|c|}{ Demographic characteristics } \\
\hline Female & $46.7(117876)$ & $45.7(244)$ & $61.8(247)$ & $59.6(339)$ \\
\hline Mean age (SD) & $73.3(13.1)$ & $64.5(13.7)$ & $73.7(14.0)$ & $73.0(11.2)$ \\
\hline \multicolumn{5}{|c|}{ Stroke risk factors and comorbidity } \\
\hline Mean $\mathrm{CHA}_{2} \mathrm{DS}_{2}$-VASc score (SD) & $3.1(1.8)$ & $2.5(1.7)$ & $3.6(2.0)$ & $3.3(1.8)$ \\
\hline Mean HAS-BLED score (SD) & $2.2(1.2)$ & $1.9(1.3)$ & $2.5(1.4)$ & $2.5(1.3)$ \\
\hline Prior stroke & $16.9(42585)$ & $14.2(76)$ & $30.3(121)$ & $20.2(115)$ \\
\hline Heart failure & $25.7(64704)$ & $30.0(160)$ & $30.3(121)$ & $29.3(167)$ \\
\hline Hypertension & 42.6 (107 332) & $26.6(142)$ & 44.5 (178) & $36.6(208)$ \\
\hline Myocardial infarction & 10.3 (26 089) & $8.2(44)$ & $11.3(45)$ & $9.3(53)$ \\
\hline Peripheral arterial disease & $7.6(19$ 266) & $6.7(36)$ & $10.0(40)$ & $9.0(51)$ \\
\hline Diabetes & $12.9(32$ 606) & $24.3(130)$ & $15.8(63)$ & $20.2(115)$ \\
\hline Prior bleeding & $26.4(66$ 694) & $28.1(150)$ & $44.0(176)$ & $37.6(214)$ \\
\hline Renal dysfunction & $5.2(13003)$ & $10.1(54)$ & $8.5(34)$ & $15.8(90)$ \\
\hline Prior venous thromboembolism & $4.8(12081)$ & $7.3(39)$ & $10.8(43)$ & $10.0(57)$ \\
\hline Chronic pulmonary disease & $14.5(36$ 615) & $28.7(153)$ & $22.3(89)$ & $30.8(175)$ \\
\hline Cancer & $15.9(40171)$ & $12.9(69)$ & $14.8(59)$ & $17.0(97)$ \\
\hline Alcohol-related disease & $4.2(10471)$ & $19.1(102)$ & $11.0(44)$ & 20.9 (119) \\
\hline \multicolumn{5}{|c|}{ Medication use within 365 days before index date } \\
\hline Coumarin & $14.4(36$ 326) & $5.1(27)$ & $8.8(35)$ & $12.1(69)$ \\
\hline NOAC & $2.5(6347)$ & $3.4(18)$ & $2.3(9)$ & $2.3(13)$ \\
\hline Aspirin & $37.6(94951)$ & $33.0(176)$ & 40.5 (162) & $38.1(217)$ \\
\hline Clopidogrel & $4.3(10936)$ & $5.6(30)$ & $9.0(36)$ & $5.6(32)$ \\
\hline NSAID & $26.7(67468)$ & $25.1(134)$ & $27.3(109)$ & $25.0(142)$ \\
\hline Digoxin & $12.4(31200)$ & $9.9(53)$ & $8.8(35)$ & $12.1(69)$ \\
\hline Non-loop diuretics & $36.2(91336)$ & $25.1(134)$ & $38.8(155)$ & $34.4(196)$ \\
\hline Loop diuretics & 25.5 (64 337) & $34.8(186)$ & $28.8(115)$ & $36.0(205)$ \\
\hline Beta-blocker & $32.2(81335)$ & $22.7(121)$ & $28.0(112)$ & $24.3(138)$ \\
\hline Calcium channel blocker & $25.5(64355)$ & $17.0(91)$ & $31.0(124)$ & $25.1(143)$ \\
\hline Renin-angiotensin inhibitor & $35.5(89621)$ & $23.8(127)$ & 37.5 (150) & $32.0(182)$ \\
\hline Statins & $24.6(62$ 166) & $23.2(124)$ & 26.0 (104) & $27.4(156)$ \\
\hline Antiepileptics & $4.0(10077)$ & $24.3(130)$ & $16.0(64)$ & 39.5 (225) \\
\hline Anticholinergics & $0.2(456)$ & $27.2(145)$ & $2.3(9)$ & $5.3(30)$ \\
\hline $\begin{array}{l}\text { Antipsychotics, lithium and anxiolytics/ } \\
\text { hypnotics }\end{array}$ & 27.1 (68 302) & $87.1(465)$ & $63.2(253)$ & $83.3(474)$ \\
\hline Antidepressants & $14.5(36516)$ & $34.8(186)$ & $78.5(314)$ & $61.0(347)$ \\
\hline
\end{tabular}

$\mathrm{CHA}_{2} \mathrm{DS}_{2}$-VASc; icongestive heart failure; hypertension; age $\geq 75$ years; diabetes mellitus; prior stroke, transient ischemic attack or thromboembolism; vascular disease, age 65-74 years; female sex; HAS-BLED, hypertension; abnormal renal function; abnormal hepatic function; prior stroke; bleeding; labile INR (not available in this study); elderly age (aspirin, clopidogrel or non-steroidal anti-inflammatory drugs; alcohol intake; NOAC, non-vitamin K oral anticoagulant; NSAID, non-steroidal anti-inflammatory drugs.

\section{Fatal thromboembolic events}

During the 5-year follow-up, 19 fatal thromboembolic events occurred in patients with AF with schizophrenia, 11 in patients with severe depression and 20 in patients with bipolar disease. Accordingly, the rate of fatal thromboembolic events per 100 person-years was substantially lower than the rate of ischaemic stroke: 1.43 for schizophrenia vs 0.52 in matched comparisons, 1.03 vs 0.82 

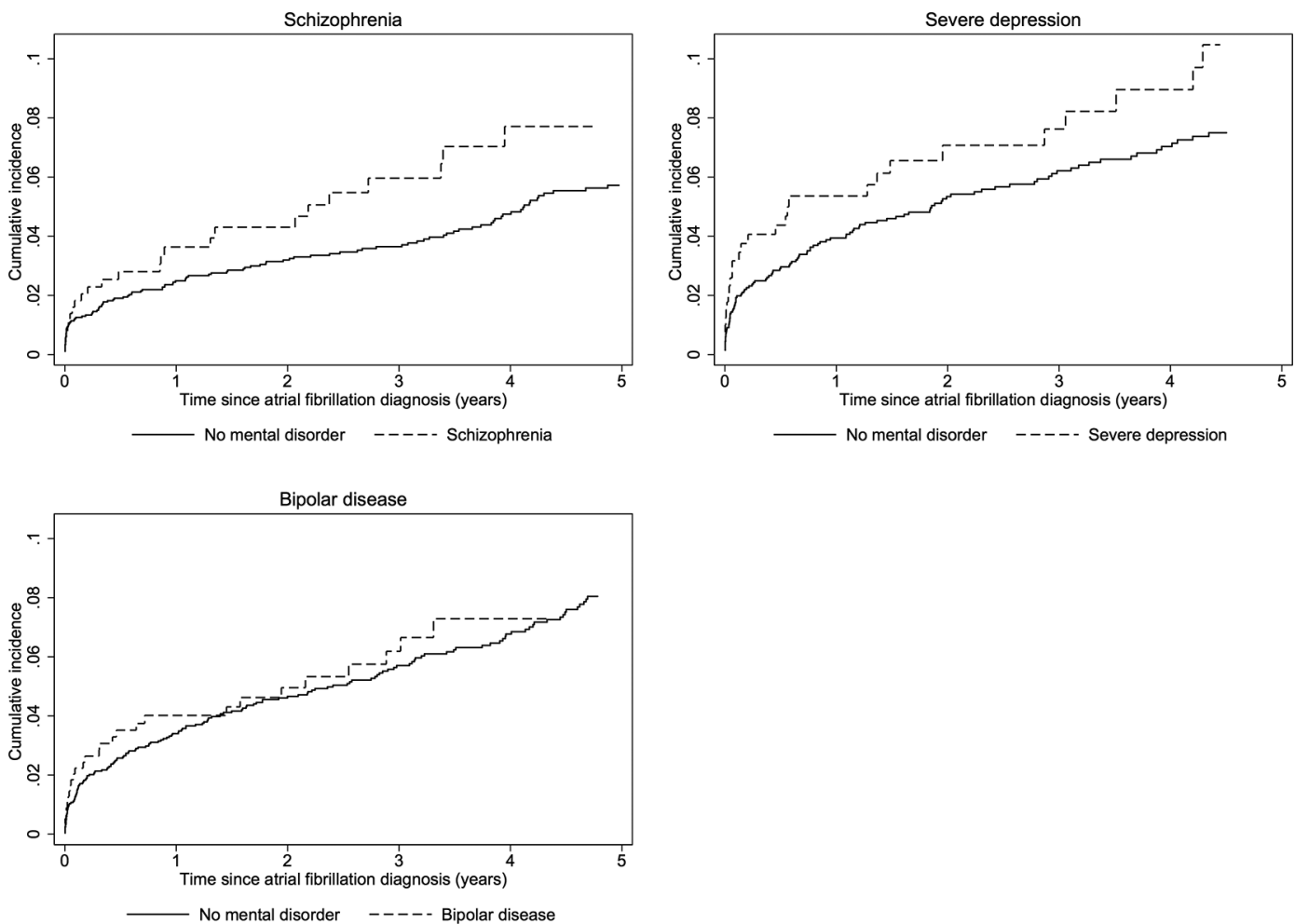

for severe depression, and 1.41 vs 0.83 for patients with bipolar disease (table 2). Notwithstanding, due to the low rate in the matched comparisons, cumulative incidence curves revealed distinct differences in rates of fatal thromboembolic events in patients with schizophrenia versus matched comparisons (figure 3 ) and to a lesser extent in patients with bipolar disease. The equivalent 5 -year HR was 3.16 (95\% CI 1.78 to 5.61 ), decreasing to 2.88 (95\% CI 1.57 to 5.28) after adjustment for stroke risk factors, comorbidity and OAT (figure 2). The unadjusted HR of fatal thromboembolic events in patients with severe depression versus matched comparisons was 1.31 (95\% CI 0.67 to 2.56). After adjustment for stroke risk factors, comorbidity and OAT, the HR was 0.75 (95\% CI 0.37 to 1.52$)$. In patients with bipolar disease the crude HR was 1.53 (95\% CI 0.93 to 2.53 ) compared with matched comparisons. Following sequential adjustment the HR was 1.23 (95\% CI 0.72 to 2.09) (figure 2).

Table 2 Number of events and rates of stroke, fatal thromboembolic events and major bleeding at 5 years following incident atrial fibrillation (AF)

\begin{tabular}{|c|c|c|c|c|c|c|c|}
\hline \multirow[b]{2}{*}{ Characteristics } & \multirow{2}{*}{$\begin{array}{l}\text { Patients } \\
\mathbf{n}\end{array}$} & \multicolumn{2}{|c|}{ Ischaemic stroke } & \multicolumn{2}{|c|}{$\begin{array}{l}\text { Fatal thromboembolic } \\
\text { events }\end{array}$} & \multicolumn{2}{|c|}{ Major bleeding } \\
\hline & & Events, $n$ & Rate & Events, $\mathrm{n}$ & Rate & Events, $\mathrm{n}$ & Rate \\
\hline \multicolumn{8}{|c|}{ Schizophrenia } \\
\hline Schizophrenia & 534 & 25 & 1.96 & 19 & 1.43 & 46 & 3.72 \\
\hline \multicolumn{8}{|c|}{ Severe depression } \\
\hline Severe depression & 400 & 28 & 2.74 & 11 & 1.03 & 41 & 4.06 \\
\hline Matched comparison cohort & 2000 & 113 & 1.93 & 50 & 0.82 & 188 & 3.24 \\
\hline \multicolumn{8}{|c|}{ Bipolar disease } \\
\hline
\end{tabular}

Rates are calculated as the number of events divided by person-time per 100 years. 


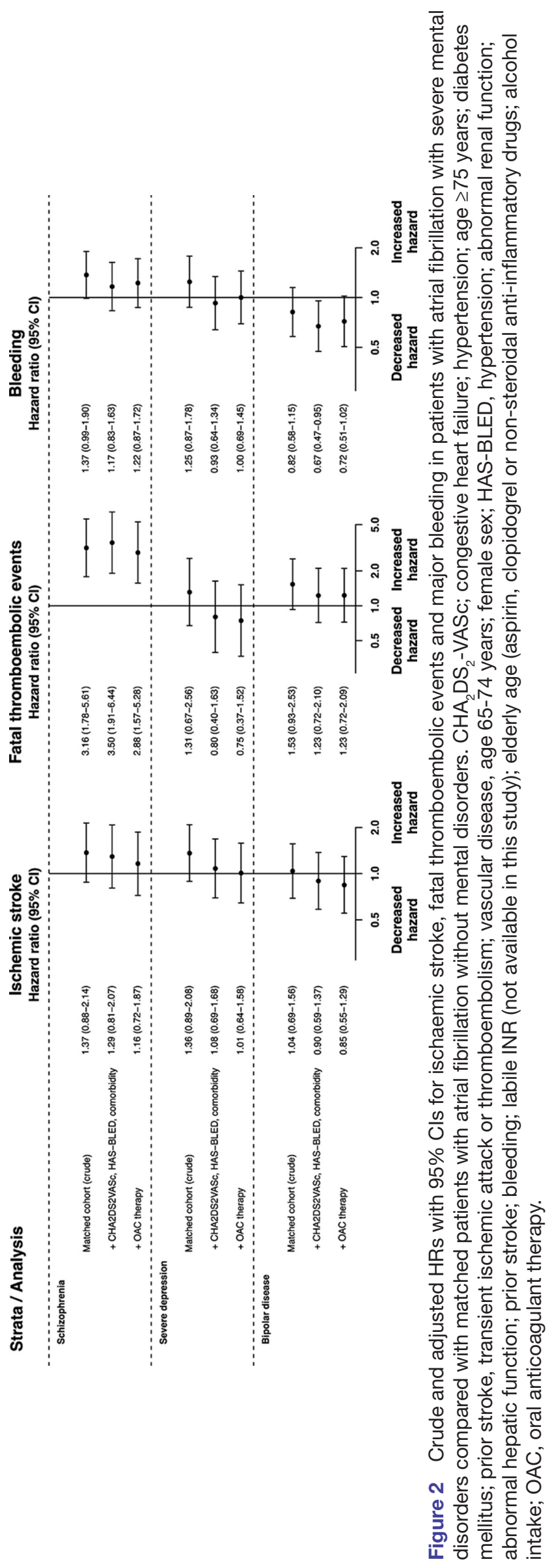

\section{Major bleeding}

The cumulative incidence curves showed higher rates of major bleeding in patients with schizophrenia and severe depression compared with their matched comparisons, whereas the curves for patients with bipolar disease overlapped with the comparisons (figure 4). At 5 years, the rate of bleeding was 3.72 events per 100 person-years in patients with schizophrenia vs 2.62 in matched comparisons (crude HR 1.37, 95\% CI 0.99 to 1.90), 4.06 per 100 person-years in patients with severe depression vs 3.24 in matched comparisons (crude HR 1.25, 95\% CI 0.87 to 1.78 ), and 2.90 vs 3.27 in patients with bipolar disease (HR $0.82,95 \%$ CI 0.58 to 1.15 ) (table 2, figure 2). Sequential adjustment for the components of the $\mathrm{CHA}_{2} \mathrm{DS}_{2}$-VASc and HAS-BLED scores (including use of aspirin, non-steroidal anti-inflammatory drugs and clopidogrel at baseline) and use of OAT attenuated the association in patients with schizophrenia and severe depression (figure 2).

\section{DISCUSSION \\ Principal findings}

This nationwide cohort study revealed a low prevalence of severe mental disorders among patients with incident AF in Denmark. Patients with schizophrenia or severe depression experienced higher risk of ischaemic stroke and major bleeding compared with matched patients with AF without mental disorders, although the differences were not statistically significant. However, after sequential adjustment for stroke and bleeding risk factors, comorbidity and use of OAT, hazard rates were comparable with matched comparisons, suggesting that the excess risk in patients with schizophrenia and severe depression derived from higher prevalence of risk factors for stroke and bleeding and differences in use of OAT. In comparison, bipolar disease was not associated with higher risk of stroke and bleeding both before and after adjustment. Few thromboembolic events occurred during follow-up. Nonetheless, we noted a substantially higher mortality after a thromboembolic event in patients with schizophrenia than in matched comparisons.

\section{Strengths and limitations}

These estimates of stroke risk and thromboembolic events in patients with $\mathrm{AF}$ are based on a nationwide cohort study conducted in a setting where virtually all medical care is provided free of charge, and with complete follow-up through nationwide registries. Nonetheless, despite universal tax-supported healthcare in Denmark, diagnoses in patients with mental disorders may have been under-reported..$^{23}$ From the current study, we are not able to conclude whether the low prevalence of patients with $\mathrm{AF}$ with mental disorders reflects the true picture or whether AF may be underdiagnosed in these patients. Likewise, we cannot exclude a possibility for differential misclassification of study outcomes, if stroke and thromboembolic events were underdiagnosed in patients with severe mental disorders. Such misclassification would bias 

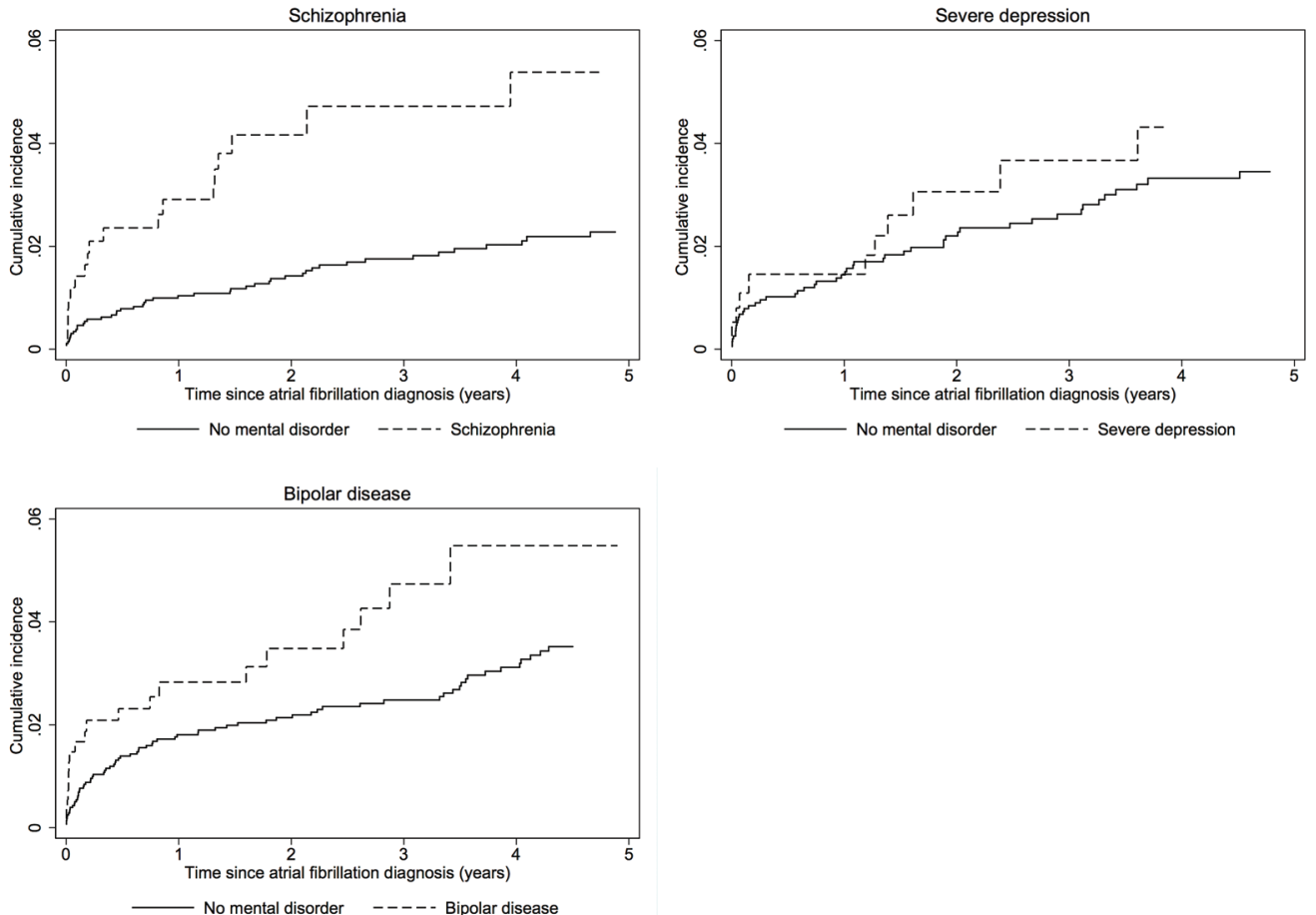

Figure 3 Cumulative incidence of fatal thromboembolic events in patients with atrial fibrillation and mental disorders and matched patients with atrial fibrillation without mental disorder.

our estimates towards the null. Furthermore, we included only patients with mental disorders recorded in the hospital register, and the prevalence is likely underestiwe infer that the majority of patients with schizophrenia and bipolar disease are in contact with the psychiatric hospital system due the severity of these conditions. It is
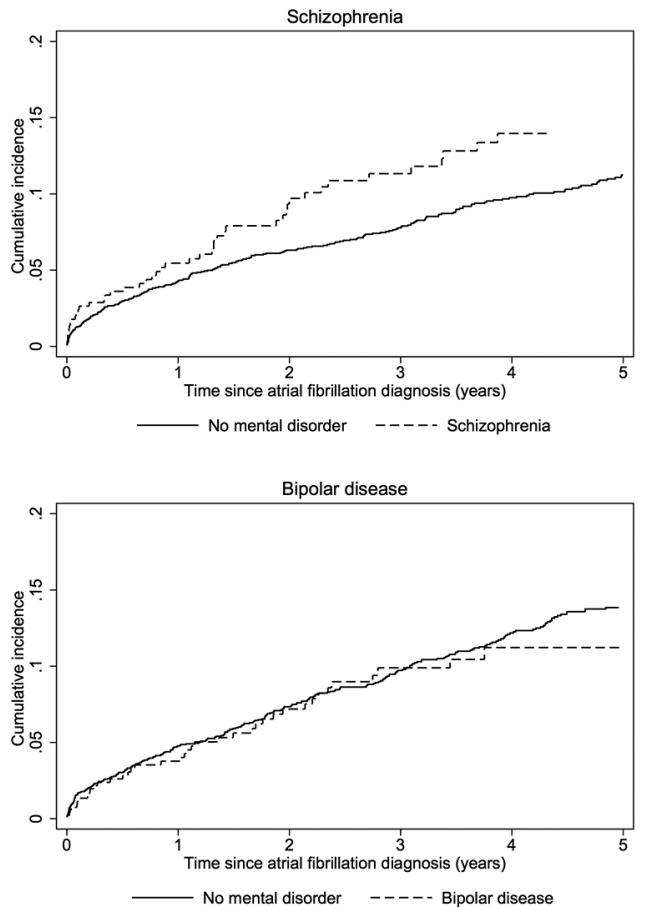
mated, mainly with regard to severe depression. However,

also important to note that we only had information on psychiatric admissions from 1995 onwards. Thus, patients with a diagnosis before 1995 and no recorded diagnosis thereafter would not be included in the exposed cohort. Lack of data on alcohol, smoking, exercise and other lifestyle-related risk factors associated with increased risk of study outcomes is another limitation. We were able to

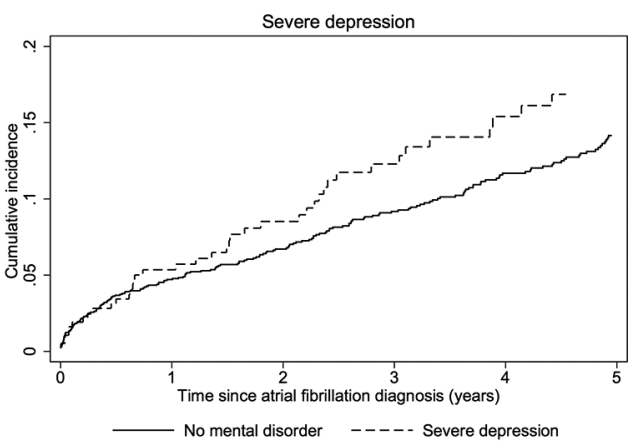
Figure 4 Cumulative incidence of major bleeding in patients with atrial fibrillation and mental disorders and matched patients
with atrial fibrillation without mental disorder. with atrial fibrillation without mental disorder. 
adjust for hospital diagnoses of alcohol-related conditions and many other lifestyle-related diseases, including chronic pulmonary disease, diabetes, liver disease, cardiovascular disease and cancer, which were more prevalent among patients with mental disorders. Nonetheless, incomplete control for these factors likely leads to residual confounding, for example, bleeding risk due to alcohol abuse. ${ }^{25}{ }^{26}$ Likewise, we did not assess the association between antipsychotic medications and stroke risk in this study. Finally, use of OAT was determined based on prescription redemption, which may be a limitation, as some patients may not take their medications. Our data did not contain information on the quality and compliance with OAT, which may be lower among patients with mental disorders due to cognitive limitations and maladaptive behaviours. ${ }^{27}$

\section{Comparison with other studies}

Our findings are concordant with two prior studies on outcomes of patients with $\mathrm{AF}$ with mental disorders receiving warfarin. ${ }^{11}{ }^{13}$ In a US cohort study of 9345 Medicaid recipients receiving two or more warfarin prescriptions less than 100 days apart, Schauer et $a l^{13}$ demonstrated HRs of 1.36 (95\% CI 1.06 to 1.74) for ischaemic stroke in patients with mental disorders compared with patients without. In another US cohort study, Paradise et $a l^{11}$ showed that mental disorders were associated with an increased risk of major bleeding (HR 1.19, 95\% CI 1.11 to 1.27 ) in patients with any mental disorder versus propensity matched comparisons in 103897 patients receiving warfarin for at least 6 months through the Veterans Health Administration. Both studies only included patients who were considered appropriate for OAT and who actually received it and remained successful on treatment for an extended period. Thus, these results may not reflect stroke risk in all patients with mental disorders.

Our findings expand prior studies by including all patients with AF regardless of OAT use. We saw a noticeable lower rate of OAT initiation in patients with mental disorders compared with matched comparisons, which is in line with prior studies showing that patients with $\mathrm{AF}$ with mental disorders are less likely to receive OAT. ${ }^{9} 10$ Schmitt $e t a l^{10}$ found that patients with AF with mental disorders had a higher prevalence of stroke risk factors and contraindications to OAT. Thus, lower treatment rates in patients with mental disorders may reflect appropriate attention to bleeding risk. On the other hand, when restricting to patients eligible for OAT, patients with mental disorders remained less likely to receive OAT. ${ }^{10}$ As the higher stroke rates in our study appeared to be due to differences in stroke risk factors, comorbidity and receipt of OAT, any disparities in the care of patients with $\mathrm{AF}$ with mental disorders require close attention. Other studies have shown that patients with mental disorders have worse OAT control. ${ }^{911} 12$ Walker et a ${ }^{9}$ found that when prescribed warfarin, patients with $\mathrm{AF}$ with mental disorders were substantially more likely to have highly supratherapeutic international normalised ratio (INR) values than those without mental disorders $(27.3 \%$ vs $1.6 \%$ had at least one INR measurement above 5.0 ). However, this assessment was based on a subcohort of only 84 patients with $\mathrm{AF}$ and should be interpreted with caution.

The higher mortality within the 30 days following a thromboembolic event in patients with schizophrenia is a concern and emphasises the need for vigilant follow-up in this patient population. The finding is in line with prior studies reporting increased cardiac mortality in patients with schizophrenia. ${ }^{24}$ Future studies are encouraged to explore potential reasons, which are likely multifactorial and may entail both severity of illness, comorbidity, quality of care and factors beyond patient care. ${ }^{29}$

\section{CONCLUSIONS}

In conclusion, this study showed that patients with $\mathrm{AF}$ with schizophrenia or severe depression were at increased risk of ischaemic stroke and major bleeding. However, the fully adjusted hazard rates were comparable with matched comparisons, indicating that the excess risk is due to a higher prevalence of risk factors for stroke and bleeding and lower use of OAT in patients with mental disorders. In comparison, rates were not increased in patients with bipolar disease. Patients with schizophrenia further experienced higher mortality following a thromboembolic event than matched comparisons. These findings highlight the importance of close attention to stroke and bleeding risk factors and potential disparities in receipt of OAT in patients with AF with mental disorders. Our findings also identify challenges in the management of patients with $\mathrm{AF}$ with mental disorders; the excess burden of stroke risk factors signifies the need for stroke prevention, whereas the higher rates of major bleeding emphasise that cautious assessment of bleeding risk and quality of OAT may be particularly pertinent in these patients. In this respect, our findings indicate a need for optimised coordination and collaboration between general somatic and mental health services.

Contributors MS, FS and JNK had full access to all of the data in this study and take responsibility for the integrity of the data and the accuracy of the data analysis. SR, SPH and TBL provided the idea for the study. MS, FS, TBL, SPH and SR defined the study concept and performed the critical interpretation of the data. MS drafted the article. All authors contributed critical revisions and approved the final version to be published.

Funding The Obel Family Foundation partly funded this research by an unrestricted grant. The sponsor had no role in the design and conduct of the study; collection, management, analysis and interpretation of the data; and preparation, review or approval of the manuscript.

Competing interests TBL has served as an investigator for Janssen Scientific Affairs and Boehringer Ingelheim, and has served as a speaker for Bayer, BMS/ Pfizer and Boehringer Ingelheim. FS has served as a consultant for Bayer. Other authors: none declared.

Patient consent Detail has been removed from this case description/these case descriptions to ensure anonymity. The editors and reviewers have seen the detailed information available and are satisfied that the information backs up the case the authors are making. 
Ethics approval The study was approved by the Danish Data Protection Agency (record number 2012-41-0633). According to Danish law, approval from an ethics committee is not required for anonymous registry-based studies.

Provenance and peer review Not commissioned; externally peer reviewed.

Data sharing statement № additional data are available.

Open Access This is an Open Access article distributed in accordance with the Creative Commons Attribution Non Commercial (CC BY-NC 4.0) license, which permits others to distribute, remix, adapt, build upon this work non-commercially, and license their derivative works on different terms, provided the original work is properly cited and the use is non-commercial. See: http://creativecommons.org/ licenses/by-nc/4.0/

(c) Article author(s) (or their employer(s) unless otherwise stated in the text of the article) 2017. All rights reserved. No commercial use is permitted unless otherwise expressly granted.

\section{REFERENCES}

1. De Hert M, Dekker JM, Wood D, et al. Cardiovascular disease and diabetes in people with severe mental illness position statement from the European Psychiatric Association (EPA), supported by the European Association for the Study of Diabetes (EASD) and the European Society of Cardiology (ESC). Eur Psychiatry 2009;24:412-24.

2. Laursen TM, Munk-Olsen T, Gasse C. Chronic somatic comorbidity and excess mortality due to natural causes in persons with schizophrenia or bipolar affective disorder. PLoS One 2011;6:e24597.

3. Bresee LC, Majumdar SR, Patten SB, et al. Prevalence of cardiovascular risk factors and disease in people with schizophrenia: a population-based study. Schizophr Res 2010;117:75-82.

4. Bernardo M, Cañas F, Banegas JR, et al. Prevalence and awareness of cardiovascular risk factors in patients with schizophrenia: a crosssectional study in a low cardiovascular disease risk geographical area. Eur Psychiatry 2009;24:431-41.

5. Osborn DP, Wright CA, Levy G, et al. Relative risk of diabetes, dyslipidaemia, hypertension and the metabolic syndrome in people with severe mental illnesses: systematic review and metaanalysis. BMC Psychiatry 2008;8:84.

6. Correll CU, Detraux J, De Lepeleire J, et al. Effects of antipsychotics, antidepressants and mood stabilizers on risk for physical diseases in people with schizophrenia, depression and bipolar disorder. World Psychiatry 2015;14:119-36.

7. Wang J, Liu YS, Zhu WX, et al. Olanzapine-induced weight gain plays a key role in the potential cardiovascular risk: evidence from heart rate variability analysis. Sci Rep 2014;4:7394.

8. Kirchhof P, Benussi S, Kotecha D, et al. ESC Guidelines for the management of atrial fibrillation developed in collaboration with EACTS. Europace 2016;2016:1609-78.

9. Walker GA, Heidenreich PA, Phibbs CS, et al. Mental illness and warfarin use in atrial fibrillation. Am J Manag Care 2011;17:617-24.

10. Schmitt SK, Turakhia MP, Phibbs CS, et al. Anticoagulation in atrial fibrillation: impact of mental illness. Am J Manag Care 2015;21:e609-e617.
11. Paradise HT, Berlowitz DR, Ozonoff A, et al. Outcomes of anticoagulation therapy in patients with mental health conditions. $J$ Gen Intern Med 2014;29:855-61.

12. Rose AJ, Hylek EM, Ozonoff A, et al. Patient characteristics associated with oral anticoagulation control: results of the Veterans AffaiRs Study to Improve Anticoagulation (VARIA). J Thromb Haemost 2010;8:2182-91.

13. Schauer DP, Moomaw CJ, Wess M, et al. Psychosocial risk factors for adverse outcomes in patients with nonvalvular atrial fibrillation receiving warfarin. J Gen Intern Med 2005;20:1114-9.

14. Schmidt M, Schmidt SA, Sandegaard JL, et al. The danish national patient registry: a review of content, data quality, and research potential. Clin Epidemiol 2015;7:449-90.

15. Pottegård A, Schmidt SAJ, Wallach-Kildemoes $\mathrm{H}$, et al. Data resource profile: the danish national prescription registry. Int $J$ Epidemiol 2017;46:dyw213.

16. Schmidt M, Pedersen L, Sørensen HT. The danish civil registration system as a tool in epidemiology. Eur J Epidemiol 2014;29:541-9.

17. Thygesen LC, Daasnes C, Thaulow I, et al. Introduction to danish (nationwide) registers on health and social issues: structure, access, legislation, and archiving. Scand J Public Health 2011;39:12-16.

18. Sundbøll J, Adelborg K, Munch T, et al. Positive predictive value of cardiovascular diagnoses in the danish national patient registry: a validation study. BMJ Open 2016;6:e012832.

19. lacus SM, King G, Porro G. Matching for causal inference without balance checking. Available SSRN 2008;1152391:1-17.

20. Blackwell M, lacus S, King G, et al. Cem: coarsened exact matching in stata. Stata J 2009;9:524-46.

21. Lip GY, Nieuwlaat R, Pisters R, et al. Refining clinical risk stratification for predicting stroke and thromboembolism in atrial fibrillation using a novel risk factor-based approach: the euro heart survey on atrial fibrillation. Chest 2010;137:263-72.

22. Pisters R, Lane DA, Nieuwlaat $R$, et al. A novel user-friendly score (HAS-BLED) to assess 1-year risk of major bleeding in patients with atrial fibrillation: the Euro heart survey. Chest 2010;138:1093-100.

23. Laursen TM, Munk-Olsen T, Agerbo E, et al. Somatic hospital contacts, invasive cardiac procedures, and mortality from heart disease in patients with severe mental disorder. Arch Gen Psychiatry 2009;66:713-20.

24. Crump C, Winkleby MA, Sundquist K, et al. Comorbidities and mortality in persons with schizophrenia: a Swedish national cohort study. Am J Psychiatry 2013;170:324-33.

25. Roth JA, Bradley K, Thummel KE, et al. Alcohol misuse, genetics, and major bleeding among warfarin therapy patients in a community setting. Pharmacoepidemiol Drug Saf 2015;24:619-27.

26. Sandén P, Renlund H, Svensson PJ, et al. Bleeding complications in venous thrombosis patients on well-managed warfarin. J Thromb Thrombolysis 2016;41:351-8.

27. Lawrence D, Kisely S. Inequalities in healthcare provision for people with severe mental illness. J Psychopharmacol 2010;24:61-8.

28. Olfson M, Gerhard T, Huang C, et al. Premature mortality among adults with schizophrenia in the United States. JAMA Psychiatry 2015;72:1172-81.

29. Ringen PA, Engh JA, Birkenaes AB, et al. Increased mortality in schizophrenia due to cardiovascular disease - a non-systematic review of epidemiology, possible causes, and interventions. Front Psychiatry 2014;5:1-11. 